\title{
Advanced neuroimaging techniques for evaluating pediatric epilepsy
}

\author{
Yun Jeong Lee, MD \\ Department of Pediatrics, Kyungpook National University Hospital, School of Medicine, Kyungpook National University, Daegu, Korea
}

Accurate localization of the seizure onset zone is important for better seizure outcomes and preventing deficits following epilepsy surgery. Recent advances in neuroimaging techniques have increased our understanding of the underlying etiology and improved our ability to noninvasively identify the seizure onset zone. Using epilepsy-specific magnetic resonance imaging (MRI) protocols, structural MRI allows better detection of the seizure onset zone, particularly when it is interpreted by experienced neuroradiologists. Ultra-high-field imaging and postprocessing analysis with automated machine learning algorithms can detect subtle structural abnormalities in MRI-negative patients. Tractography derived from diffusion tensor imaging can delineate white matter connections associated with epilepsy or eloquent function, thus, preventing deficits after epilepsy surgery. Arterial spin-labeling perfusion MRI, simultaneous electroencephalography (EEG)-functional MRI (fMRI), and magnetoencephalography (MEG) are noinvasive imaging modalities that can be used to localize the epileptogenic foci and assist in planning epilepsy surgery with positron emission tomography, ictal single-photon emission computed tomography, and intracranial EEG monitoring. MEG and fMRI can localize and lateralize the area of the cortex that is essential for language, motor, and memory function and identify its relationship with planned surgical resection sites to reduce the risk of neurological impairments. These advanced structural and functional imaging modalities can be combined with postprocessing methods to better understand the epileptic network and obtain valuable clinical information for predicting long-term outcomes in pediatric epilepsy.

Key words: Child, Epilepsy, Neuroimaging, Diffusion tensor imaging, Functional magnetic resonance imaging

\section{Introduction}

Epilepsy is the most common chronic neurologic disorder in the pediatric population. The incidence of epilepsy in childhood is approximately $40-100$ cases per 100,000 individuals per year, with the highest incidence in the first year of life. ${ }^{1,2}$ Although the long-term outcome is reasonably favorable with nearly twothirds of patients in terminal remission, approximately one-third of children have drug-resistant epilepsy. ${ }^{3)}$ Among these patients, especially those with focal epilepsy, surgical treatment aiming to remove the epileptogenic foci may be the only available option to achieve a seizure-free status. The role of neuroimaging in epilepsy is detecting structural abnormalities that may be related to epilepsy and provide information for acute and long-term treatment planning and about prognosis. ${ }^{4,5)}$ Recent advances in neuroimaging techniques have allowed the possibility of defining the subtle structural abnormalities that might cause epilepsy and predicting and reducing neurologic impairment after surgical resection. Furthermore, methodologies to define functional connectivity, which may be related to the comorbidity of epilepsy and seizure outcome, have also rapidly progressed.

In this review, we explore the recent developments in neuroimaging techniques for evaluating pediatric epilepsy with a focus on the acquisition method, postprocessing analysis, and multidisciplinary approach using various neuroimaging modalities. We divide these tools into structural neuroimaging, which examines the structure of the nervous system, and functional neuroimaging, which measures an aspect of human brain function based on localized changes in brain metabolism related to neural activity.

\section{Structural neuroimaging in epilepsy}

Although computed tomography (CT) can rapidly rule out an intracranial hemorrhage or a mass requiring urgent neurosurgical attention, magnetic resonance imaging (MRI) has become an essential imaging modality to identify epileptogenic lesions because of its superior depiction of the brain anatomy and tissue composition. ${ }^{6}$ Patients with a normal CT scan should undergo further evaluations since MRI can identify certain epileptogenic lesions in $8 \%-12 \%$ of patients with normal CT results. ${ }^{7-9)}$ Never-

\footnotetext{
Corresponding author: Yun Jeong Lee, MD. Department of Pediatrics, Kyungpook National University Hospital, 130, Dongdeok-ro, Jung-gu, Daegu 41944, Korea 凶 E-mail: oilily1103@hanmail.net, https://orcid.org/0000-0003-3472-5336

Received: 28 July, 2019, Revised: 3 November, 2019, Accepted: 6 November, 2019

This is an open-access article distributed under the terms of the Creative Commons Attribution Non-Commercial License (http://creativecommons.org/licenses/by$\mathrm{nc} / 4.0 /$ ) which permits unrestricted non-commercial use, distribution, and reproduction in any medium, provided the original work is properly cited.

Copyright (c) 2020 by The Korean Pediatric Society
} 
theless, subtle abnormalities such as a malformation of cortical development and hippocampal sclerosis are often not identified with the current resolution and standard MRI protocol. Finally, a diagnosis is often confirmed in postoperative histopathology that was not detected on MRI. ${ }^{10)}$

The sensitivity of MRI examinations can be improved using epilepsy-specific MRI protocols interpreted by neuroradiologists with the awareness of clinical focus hypotheses. Von Oertzen et al. ${ }^{11)}$ found that the sensitivity of "nonexpert" reports of standard MRI for focal lesions, "expert" reports of standard MRI, and epilepsy-dedicated MRI were 39\%, 50\%, and 91\%, respectively. These results suggest that patients with drug-resistant epilepsy showing negative results on a standard MRI protocol should undergo MRI using a dedicated epilepsy protocol, and more training is needed to increase neuroradiologist awareness of a clinical focus hypothesis. Epilepsy-specific MRI protocols have been proposed in several studies. ${ }^{12-14)}$ A study of 2,740 surgical patients with epilepsy determined "essential 6" sequences for the detection of virtually all common epileptogenic lesion entities (Table 1). ${ }^{12)}$ An epilepsy-specific MRI protocol generally includes 3-dimensional (3D) T1-weighted volumetric acquisition, T2weighted images, fluid-attenuated inversion recovery (FLAIR), and hemosiderin- and calcium-sensitive sequences such as gradient echo (GRE) and susceptibility weighted imaging (SWI). GRE and SWI sequences are useful for detecting posttraumatic changes, remote hemorrhage, multiple cavernomas, or calcification in cortical tubers and subependymal nodules in tuberous sclerosis or calcification in low-grade tumors. The 3D T1-weighted volumetric acquisition using 1-mm isotropic voxels enables image reconstruction in any plane and allows the excellent assessment of cortical thickness and delineation between the gray-white matter, particularly in focal cortical dysplasia (FCD). ${ }^{15)}$ Furthermore, using these sequences, a variety of postprocessing techniques such as volumetric and morphometric analyses are available. ${ }^{16)}$ An epilepsy-specific MRI protocol must include

Table 1. Epilepsy outpatient-specific magnetic resonance imaging protocol: the "essential 6" sequences

\begin{tabular}{llll}
\hline Sequence & $\begin{array}{c}\text { Slice thickness } \\
\text { (no gap) }\end{array}$ & $\begin{array}{c}\text { Cut-plane } \\
\text { orientation }\end{array}$ & $\begin{array}{c}\text { Cut-plane } \\
\text { angulation }\end{array}$ \\
\hline $3 \mathrm{D}-\mathrm{T} 1$ & $1 \mathrm{~mm}$ isotropic & $3 \mathrm{D}$ & $\mathrm{AC}-\mathrm{PC}$ \\
T2/STIR & $\leq 3 \mathrm{~mm}$ & Axial & $\mathrm{HC}$ \\
T2/ STIR & $\leq 3 \mathrm{~mm}$ & Coronal & $\mathrm{HC}$ \\
FLAIR & $\leq 3 \mathrm{~mm}^{\text {a) }}$ & Axial & $\mathrm{HC}$ \\
FLAIR & $\leq 3 \mathrm{~mm}^{\text {a) }}$ & Coronal & $\mathrm{HC}$ \\
Hemo/calc & $\leq 3 \mathrm{~mm}^{\text {m }}$ & Axial & $\mathrm{HC}^{\text {b) }}$ \\
\hline
\end{tabular}

The above protocol is for a 1.5-T scanner. On a 3-T scanner, the slice thickness can be further decreased. The field of view of all sequences must cover the entire brain.

3D-T1, 3-dimensional T1; AC-PC, anterior commissure-posterior commissure; STIR, short T1 inversion recovery; HC, hippocampus; FLAIR, fluid-attenuated inversion recovery; Hemo/calc, hemosiderin- and calcium-sensitive.

a) $1-\mathrm{mm}$ isotropic 3D-FLAIR. ${ }^{\text {b) }}$ The ideal angulation of axial hemo-calc sequences is subject to further investigation.

Adapted from Wellmer et al. Epilepsia 2013;54:1977-87, with permission from John Wiley and Sons. ${ }^{12)}$ several cut-plane orientations, i.e., standard axial, coronal, and sagittal planes, to prevent the misinterpretation of partial volume effects or oddly cut physiologic structures. An acquisition plane perpendicular to the long axis of the hippocampus could improve the detection of hippocampal sclerosis and temporal encephaloceles, which are often missed in other planes. The routine administration of gadolinium contrast provides little advantage in children with new-onset epilepsy; nevertheless, contrast should be given in cases of suspected tumors, inflammation, infection, or vascular malformation. ${ }^{12,14)}$

A special sequence should be considered for patients younger than 2 years of age because of immature myelination. In infants younger than 1 year of age, FLAIR and proton density sequences are less helpful due to inadequate myelin maturation. However, it should be replaced by high-resolution T2-weighted and 3D T1-weighted (less useful $<1$ year) images. ${ }^{17)}$ In addition, FCD shows various characteristics depending on the developmental stage of myelination of the lesion and surrounding brain. In patients under 2 years of age, some FCD lesions may show a high T2 or FLAIR signal and a lower T1 signal due to secondary demyelination, dysmyelination, or gliosis. ${ }^{18)}$ Other lesions show lower T2 and higher T1 signals, which are thought to be secondary to early myelination and subsequently disappear as myelination progresses. ${ }^{19,20)}$ In cases of negative MRI findings during infancy, repeating imaging is recommended at 6-month intervals or after 24 months when more mature myelination can reveal otherwise unsuspected cortical dysplasia. ${ }^{14,19)}$ Similarly, lesions such as cortical tubers in tuberous sclerosis and Sturge-Weber syndrome may be more evident on follow-up imaging.

\section{Advances in structural neuroimaging}

\section{Ultra-high-field MRI}

The detection rate of subtle epileptic lesions is also affected by the magnetic field strength. Three Tesla (3 T) MRI is thought to be more sensitive to subtle epileptic lesions in some patients who are MRI-negative at $1.5 \mathrm{~T}^{21-23)}$ especially when phased array surface coils are used. ${ }^{24)}$ Furthermore, the use of ultra-highfield (7 T) MRI has increased for in vivo brain imaging, offering a higher signal-to-noise ratio (SNR) and contrast-to-noise ratio, which can result in higher spatial resolution. Compared with conventional field-strength MRI (1.5-3 T), the diagnostic value of 7 T MRI has been demonstrated for polymicrogyria, ${ }^{25)}$ moyamoya disease, ${ }^{26)}$ hippocampal sclerosis, ${ }^{27)}$ brain tumors, ${ }^{28)}$ stroke, ${ }^{29)}$ and multiple sclerosis (Fig. 1). ${ }^{30)}$ In a prospective study, 7 T MRI was performed for 21 consecutive patients (17 adults, 4 children) with a single seizure onset zone who were previously considered MRI-negative. GRE and FLAIR images revealed a distinct lesion in $29 \%$ of patients ( 1 child), with the histopathological diagnosis of FCD in all patients who underwent surgical resection. ${ }^{31)}$ Nevertheless, there are few reports of the clinical application of 7 T MRI in pediatric epilepsy. ${ }^{31,32)}$ High cost, increased susceptibility artifacts, image inhomogeneity, and patient 
comfort and safety problems including increased radiofrequency power deposition, dizziness, nausea, and metallic taste must be considered when using ultra-high-field MRI in clinical practice for pediatric epilepsy. ${ }^{33)}$

\section{Diffusion tensor imaging}

Diffusion tensor imaging (DTI) has been reported to better visualize the white matter fiber tracts by estimating the direction, orientation, and anisotropy of the white matter fiber tract based on the differential mobility of water molecules along versus perpendicular to the fiber bundles. DTI tractography can delineate 3D-specific white matter tracts from the preferential selection of anisotropic water diffusion by connecting fibers that pass through anatomically placed regions of interest. This technique can be useful for evaluating white matter tract alterations associated with epilepsy. ${ }^{34,35)}$ It can also be used as a presurgical evaluation tool to avoid eloquent pathways such as the corticospinal tract for motor function, optic radiation for conveying visual information, or arcuate fasciculus for language, particularly in children for whom invasive presurgical evaluation is challenging (Fig. 2). ${ }^{36-38)}$ DTI has also been used to visualize the functional connectivity of seizure tracts by developing a topographical map of seizure network nodes. ${ }^{39)}$ However, tractography has its limitations, i.e., it tends to have high variability and low reliability depending on technical factors such as data acquisition, fiber modeling, fiber reconstruction, and expert interpretation including choices of seed regions and stopping thresholds. ${ }^{40)}$ In addition, in tractography, each voxel is considered as a single diffusion compartment even though it could be a combination of white matter, gray matter, and cerebrospinal fluid (CSF). The
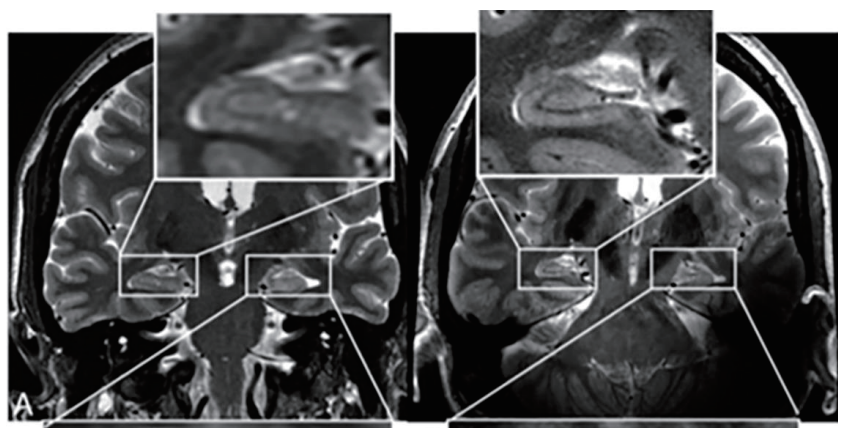

3T
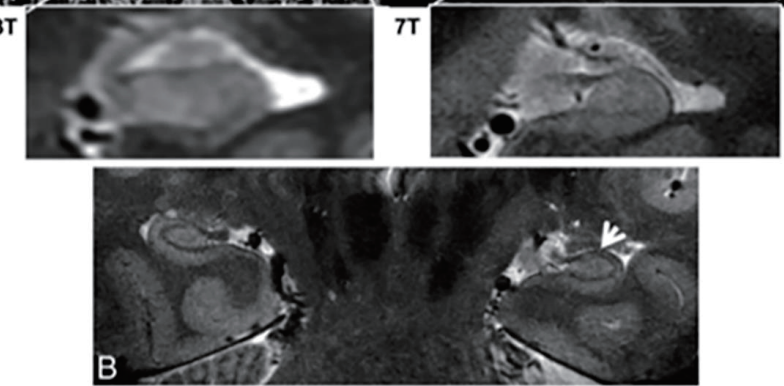

Fig. 1. (A) Comparison of $3 \mathrm{~T}$ and $7 \mathrm{~T}$ coronal fast spin-echo images in patients with mesial temporal lobe epilepsy. Subtle left hippocampal abnormalities are visualized in greater detail in the $7 \mathrm{~T}$ image. (B) In a second patient with mesial temporal lobe epilepsy, a loss of gray matter in the left hippocampus is detected on $7 \mathrm{~T}$ (white arrow) but not $3 \mathrm{~T}$ images. Adapted from Balchandani and Naidich. AJNR Am J Neuroradiol 2015;36:1204-15. ${ }^{33)}$ neurite orientation dispersion and density imaging (NODDI) technique were recently proposed to overcome this limitation. This technique assumes that each voxel is a combination of intracellular, extracellular, and CSF components. This model allows the determination of individual contributions of free water (CSF), neurite density (axons and dendrites), and orientation dispersion (myelination) for fractional anisotropy, one of the parameters of DTI. In a preliminary study, NODDI was used to detect subtle FCD by identifying reduced intracellular volume fraction, a marker of neural density, in the FCD lesion (Fig. 3). ${ }^{41}$

\section{Postprocessing of structural neuroimaging in nonlesional epilepsy}

Although a visual inspection of high-resolution MRI can improve the detection of subtle epileptogenic lesions such as hippocampal sclerosis and FCD, a conventional visual analysis has limited ability to recognize the presence and extension of subtle lesions. Advanced MRI postprocessing techniques have been developed to identify subtle structural abnormalities by quantifying the anatomic features of the brain without the need for time-consuming manual measurements or subjective visual assessments.

Voxel-based morphometry (VBM), the most popular morphometric technique to date, can be used for T1-weighted images to extract gray matter and white matter maps for comparison with a normal control database. The use of VBM has indicated that lesions with increased gray matter concentration are in agreement with FCD lesions visible on MRI in $63 \%-87 \%$ of
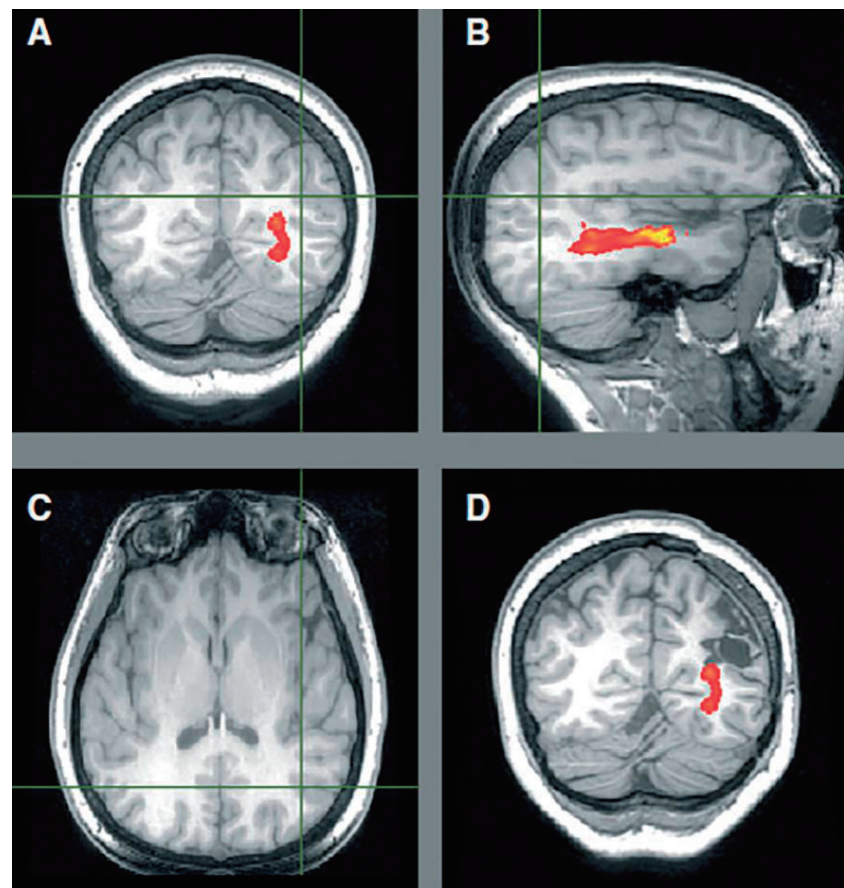

Fig. 2. Optic radiation tractography. (A-C) Preoperative tractography showing the optic radiation passing inferomedially to the left parietal focal cortical dysplasia (crosshair) on a T1-weighted image. (D) Postoperative T1-weighted image with overlaid preoperative tractography showing no damage. Visual fields are normal. Adapted from Winston et al. Epilepsia 2011;52:1430-8. ${ }^{36}$ 


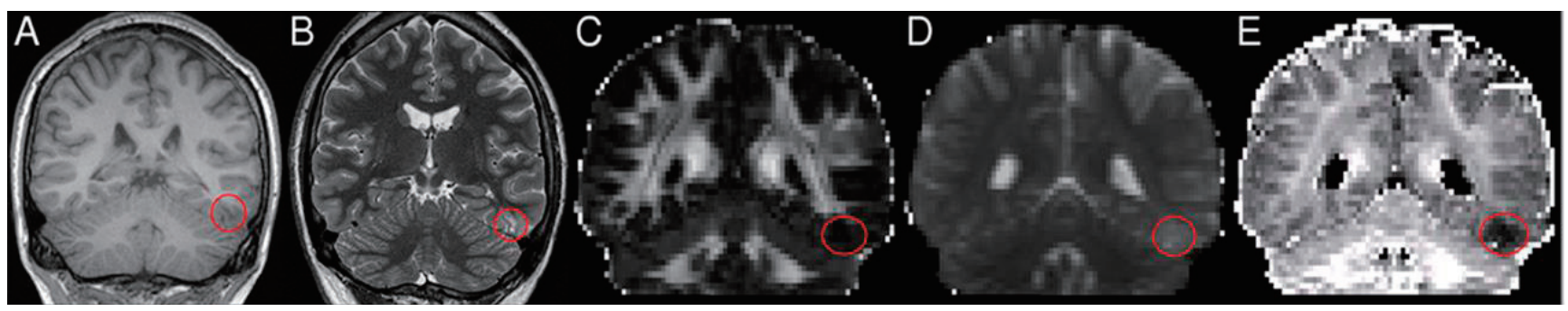

Fig. 3. Neurite orientation dispersion and density imaging (NODDI) for the detection of focal cortical dysplasia (FCD). The FCD (circle) is defined poorly on volumetric T1- (A) and T2-weighted coronal oblique (B) images and on standard diffusion images including fractional anisotropy (C) and mean diffusivity (D) maps. However, it is clearly visible as reduced intracellular volume fraction on NODDI (E). Adapted from Winston et al. Epilepsy Res 2014;108:336-9. ${ }^{41)}$

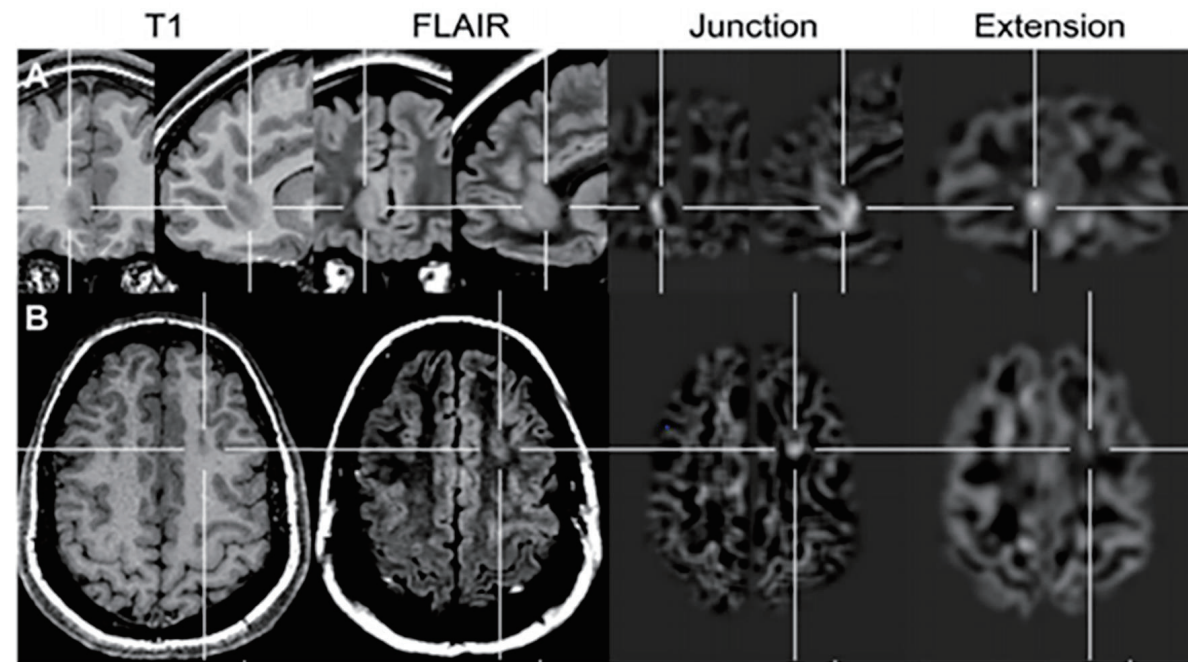

Fig. 4. Application of a voxel-based image postprocessing method. (A) Right frontomesial focal cortical dysplasia (FCD) was not detected by conventional visual analysis; however, it was clearly visible on the junction image (blurred gray-white matter junction) and extension image (gray matter extending abnormally into the white matter). (B) FCD lla located at the bottom of the left superior frontal sulcus with a moderate abnormality visible on the junction image; however, no abnormality was visible on the extension, T1-weighted, or fluid-attenuated inversion recovery images. Adapted from Wagner et al. Brain 2011;134(Pt 10):2844-54, with permission from Oxford University Press. ${ }^{45}$

cases. $^{42-44)}$ VBM also allows the enhanced visualization of FCD with a junction map to highlight blurring of the gray-white matter boundary and an extension map to delineate abnormally deep sulci (Fig. 4). ${ }^{45)}$ Surface-based morphometry techniques reconstruct the cortical surface and allow the measurement of morphologic features such as cortical thickness, curvature, and depth and texture features (blurring of the gray-white matter boundary) to detect the abnormal gyral and sulcal patterns of FCD that could not be detected on VBM. ${ }^{46}$ ) T2 relaxometry refers to the quantitative measurement of $\mathrm{T} 2$ relaxation times in the brain. This method is useful for detecting signal changes in hippocampal sclerosis in combination with hippocampal atrophy detected by automated volumetry in temporal lobe epilepsy. ${ }^{47,48)}$

Various methods of structural postprocessing analysis have been described in detail in the literature. ${ }^{16,49)}$ Hong et al..$^{50)}$ recently reported that an automated classifier relying on surfacebased features of FCD morphology and intensity detected FCD type II in 14 of $19 \mathrm{MRI}$-negative patients with 74\% sensitivity and $100 \%$ specificity. In the future, the automated detection of subtle lesions by morphometry and machine learning methods with structural imaging data showed a promising role in presurgical decision-making about the epileptogenic zone in patients previously regarded as MRI-negative. ${ }^{51)}$

\section{Functional neuroimaging in epilepsy}

\section{Localization of epileptic activity}

In patients without definite abnormalities on conventional MRI, functional neuroimaging may aid our understanding of the mechanisms underlying the epileptic process, leading to a more accurate localization of abnormal neuronal activities by measuring in vivo perfusion, metabolic changes, or neurotransmission abnormalities associated with the seizure. In fact, 18F-fluorodeoxyglucose (FDG)-positron emission tomography (PET) is the most widely used modality for detecting focal areas of relative hypometabolism, which may reflect functional disturbances in cerebral activity associated with the epileptogenic zone. The regional cerebral hypometabolism identified by FDG-PET often has a wider distribution than that of the seizure focus, which can 


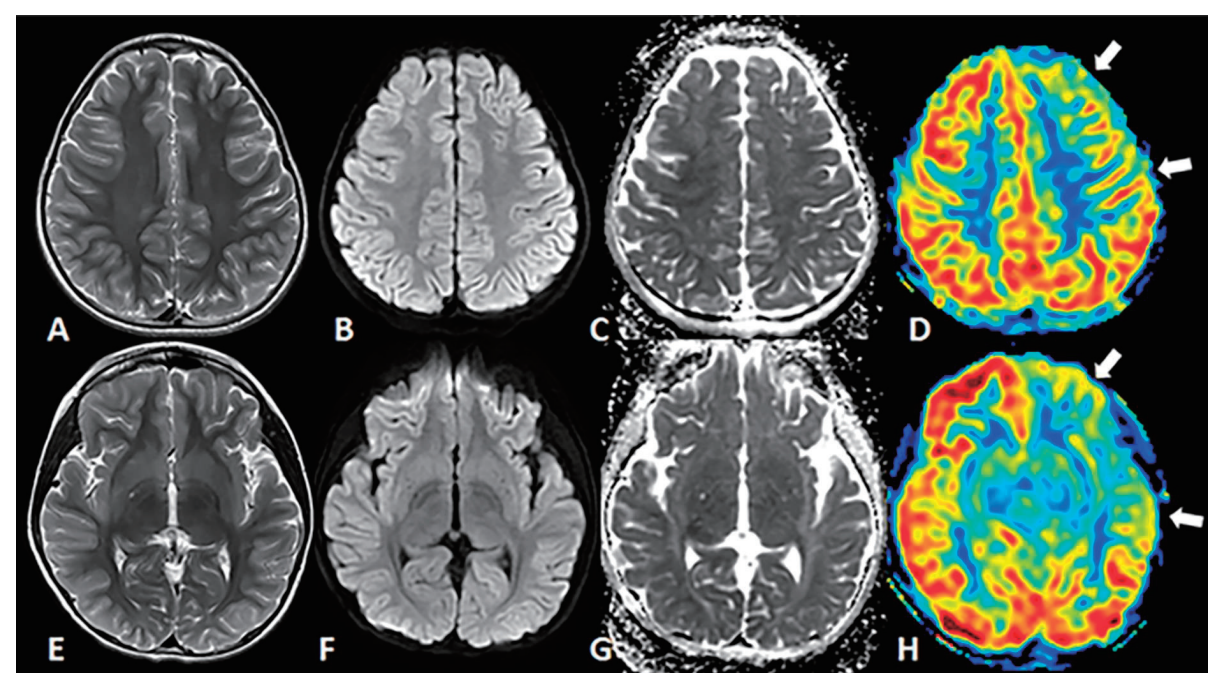

Fig. 5. Arterial spin labeling (ASL) perfusion magnetic resonance image (MRI) taken at 1 day after seizure onset. A 7-year-old girl presented with a focal dyscognitive seizure. Her interictal electroencephalography (EEG) results were normal. Axial T2-weighted images ( $A, E)$, diffusion-weighted images $(B, F)$, and apparent diffusion coefficient images $(C, G)$ showed no abnormal focal lesions in the brain parenchyma. ASL perfusion MRI $(D, H)$ revealed hypoperfusion in the left frontotemporal lobes (arrows). In this patient, the clinical seizure focus was in the left frontotemporal area. Reprinted from Lee et al. Seizure 2019;65:151-8, with permission from Elsevier Ltd. ${ }^{59}$

represent the focus and the projection areas of seizure activity. ${ }^{52)}$ This could determine the extent of resection difficulty. FDGPET is also a useful tool for predicting seizure control after epilepsy surgery; the extent of resection of the hypometabolism on FDG-PET may be associated with better a postoperative seizure outcome..$^{53-55)}$

Single-photon emission computed tomography (SPECT) is a nuclear imaging modality that can show the cerebral perfusion before, during, and after a seizure using a radioactive tracer. To increase its sensitivity, the subtraction of interictal from ictal SPECT coregistered to MRI techniques is used to complement visual interpretation to identify subtle seizure-associated changes. However, the delayed injection of the tracer and a short seizure duration can result in a variable pattern of perfusion changes according to the seizure propagation rather than the seizure onset zone. Therefore, the use of PET or SPECT in the peri-ictal period is often difficult in the pediatric population due to the requirement for a separate evaluation and administration of radiopharmaceuticals.

Arterial spin labeling (ASL) is a perfusion MRI technique that assesses cerebral blood flow by magnetically labeling inflowing blood water as an endogenous tracer ${ }^{56}$ By comparing the signal difference between labeled and control images, ASL can identify peri-ictal perfusion abnormalities and provide complementary information about the seizure onset zone. The perfusion map obtained from ASL corresponds well to PET imaging and electroencephalography (EEG) results of intractable epilepsy. ${ }^{57,58)}$ Although ASL suffers from a relatively low SNR, advantages such as noninvasiveness, easy accessibility, high reproducibility, and the absence of intravenous contrast agents make it particularly suitable for pediatric epilepsy. In our study, 24 of 36 (66.7\%) children with focal seizures showed ASL abnormalities during the post- or interictal periods; of them, 19 (52.8\%) showed con- cordance between ASL and clinical seizure focus (Fig. 5). ${ }^{59)}$

\section{Mapping of eloquent brain function}

Functional MRI (fMRI) is based on the principle that metabolically active regions of the brain have increased blood flow, known as activation-flow coupling. It is used to identify blood oxygenation level-dependent (BOLD) signals, which are determined by the local concentration of deoxygenated and oxygenated hemoglobin in response to neural activities. Recently, fMRI has become a promising noninvasive alternative technique to the Wada test (intracarotid amobarbital test) in the adult population to guide the resection plan by identifying critical areas of the brain near the seizure focus and minimize the risk of new deficits. ${ }^{60)}$ The patient is asked to perform a series of tasks during a task-based fMRI study. Although strong preliminary evidence shows that fMRI can be used to lateralize and localize language and motor function in pediatric epilepsy surgery candidates, functional mapping via task-based $\mathrm{fMRI}$ is challenging in children due to a lack of cooperation, developmental immaturity, and age-dependent cerebral plasticity. ${ }^{61)}$ Furthermore, it remains unclear which language and memory tasks produce optimal activation and how it is statistically quantified. Further studies specifically targeting pediatric epilepsy populations are needed. Resting-state fMRI has been used to localize the eloquent cortex by identifying specific brain resting-state networks for presurgical planning in patients who may not be able to cooperate in a taskbased paradigm. ${ }^{62,63)}$

\section{Advances in functional neuroimaging}

Functional MRI is a hemodynamic imaging method that lacks the temporal resolution of EEG. The combination of EEG 
with fMRI (EEG-fMRI) allows the detection of BOLD signal changes related to ictal or interictal epileptic discharges (IEDs). EEG-fMRI has been used to follow propagation of the seizure and seizure foci in real-time across the brain and finally delineate the epileptogenic network of focal and generalized epilepsy. ${ }^{64)}$ Furthermore, functional connectivity analyses using EEG-fMRI can identify the real-time effect of IEDs on cognition ${ }^{65)}$ and predict the surgical outcome of epilepsy ${ }^{66}$ However, collecting EEG-fMRI recordings in some children is challenging due to a lack of cooperation, and various factors including sedation, age, sleep, and the effect of antiepileptic drugs on hemodynamic parameters should be further investigated. ${ }^{64}$

Magnetoencephalography (MEG) records the magnetic fields generated by synchronized neural activities of the brain with excellent spatial and temporal resolution. The strength of MEG is that the magnetic field flux is not distorted by muscle artifacts or materials that lie between the brain and recording devices. MEG has been used to detect the exact location of sources and superposition them on brain MRI, known as magnetic source imaging. Its accuracy for localizing the irritative zone is comparable to electrocorticography, ictal SPECT, or PET. ${ }^{67-69)}$ However, it has limitations for detecting neural activities from deeper sources with a radial orientation; nevertheless, its combined use with EEG could improve the accuracy of source localization and identification of propagated activity. ${ }^{70)}$ In addition, MEG functional mapping is promising for pediatric patients who cannot undergo intraoperative cortical mapping. ${ }^{71)}$

\section{Conclusion}

In recent years, the field of neuroimaging has undergone considerable development. Although many of the imaging techniques described here are still considered preliminary, the increased availability of ultra-high-field imaging, advanced imaging technology, and postprocessing analysis will improve the detection of subtle lesions that underlie refractory focal epilepsy. A combined approach using several structural and functional neuroimaging datasets in a machine learning model may play an essential role in noninvasively localizing the epileptogenic foci for possible surgical resection. Furthermore, functional neuroimaging modalities such as fMRI and MEG can be used as alternatives to the Wada test to elucidate eloquent structures for preventing postoperative neurologic deficits. Continued advances in neuroimaging will enhance our understanding of the epileptic network and guide therapeutic strategies to achieve better outcomes.

\section{Conflict of interest}

No potential conflicts of interest relevant to this article are reported.

\section{Acknowledgments}

This study was supported by the National Research Foun- dation of Korea (NRF-2018R1C1B5085985).

See the commentary "Guideline for advanced neuroimaging in pediatric epilepsy" in Volume 63 on page 100.

\section{References}

1. Hauser WA. The prevalence and incidence of convulsive disorders in children. Epilepsia 1994;35 Suppl 2:S1-6.

2. Wirrell EC, Grossardt BR, Wong-Kisiel LC, Nickels KC. Incidence and classification of new-onset epilepsy and epilepsy syndromes in children in Olmsted County, Minnesota from 1980 to 2004: a population-based study. Epilepsy Res 2011;95:110-8.

3. Sillanpää M, Schmidt D. Natural history of treated childhood-onset epilepsy: prospective, long-term population-based study. Brain 2006; 129(Pt 3):617-24.

4. Semah F, Picot MC, Adam C, Broglin D, Arzimanoglou A, Bazin B, et al. Is the underlying cause of epilepsy a major prognostic factor for recurrence? Neurology 1998;51:1256-62.

5. Garcia Gracia C, Yardi R, Kattan MW, Nair D, Gupta A, Najm I, et al. Seizure freedom score: a new simple method to predict success of epilepsy surgery. Epilepsia 2015;56:359-65.

6. Bronen RA, Fulbright RK, Spencer DD, Spencer SS, Kim JH, Lange RC, et al. Refractory epilepsy: comparison of MR imaging, CT, and histopathologic findings in 117 patients. Radiology 1996;201:97-105.

7. King MA, Newton MR, Jackson GD, Fitt GJ, Mitchell LA, Silvapulle MJ, et al. Epileptology of the first-seizure presentation: a clinical, electroencephalographic, and magnetic resonance imaging study of 300 consecutive patients. Lancet 1998;352:1007-11.

8. Ho K, Lawn N, Bynevelt M, Lee J, Dunne J. Neuroimaging of first-ever seizure: Contribution of MRI if CT is normal. Neurol Clin Pract 2013; 3:398-403

9. Resta M, Palma M, Dicuonzo F, Spagnolo P, Specchio LM, Laneve A, et al. Imaging studies in partial epilepsy in children and adolescents. Epilepsia 1994;35:1187-93.

10. Bien CG, Szinay M, Wagner J, Clusmann H, Becker AJ, Urbach H. Characteristics and surgical outcomes of patients with refractory magnetic resonance imaging-negative epilepsies. Arch Neurol 2009;66:1491-9.

11. Von Oertzen J, Urbach H, Jungbluth S, Kurthen M, Reuber M, Fernández $\mathrm{G}$, et al. Standard magnetic resonance imaging is inadequate for patients with refractory focal epilepsy. J Neurol Neurosurg Psychiatry 2002;73: 643-7.

12. Wellmer J, Quesada CM, Rothe L, Elger CE, Bien CG, Urbach H. Proposal for a magnetic resonance imaging protocol for the detection of epileptogenic lesions at early outpatient stages. Epilepsia 2013;54:197787.

13. Cendes F. Neuroimaging in investigation of patients with epilepsy. Continuum (Minneap Minn) 2013;19(3 Epilepsy):623-42.

14. Gaillard WD, Chiron C, Cross JH, Harvey AS, Kuzniecky R, HertzPannier L, et al. Guidelines for imaging infants and children with recentonset epilepsy. Epilepsia 2009;50:2147-53.

15. Montenegro MA, Li LM, Guerreiro MM, Guerreiro CA, Cendes F. Focal cortical dysplasia: improving diagnosis and localization with mag. netic resonance imaging multiplanar and curvilinear reconstruction. J Neuroimaging 2002;12:224-30.

16. Martin P, Bender B, Focke NK. Post-processing of structural MRI for individualized diagnostics. Quant Imaging Med Surg 2015;5:188-203.

17. Vézina LG. MRI-negative epilepsy: protocols to optimize lesion detection. Epilepsia 2011;52 Suppl 4:25-7.

18. Yagishita A, Arai N, Maehara T, Shimizu H, Tokumaru AM, Oda M. Focal cortical dysplasia: appearance on MR images. Radiology 1997;203:5539.

19. Daghistani R, Widjaja E. Role of MRI in patient selection for surgical treatment of intractable epilepsy in infancy. Brain Dev 2013;35:697-705. 
20. Eltze CM, Chong WK, Bhate S, Harding B, Neville BG, Cross JH. Taylortype focal cortical dysplasia in infants: some MRI lesions almost disappear with maturation of myelination. Epilepsia 2005;46:1988-92.

21. Nguyen DK, Rochette E, Leroux JM, Beaudoin G, Cossette P, Lassonde $\mathrm{M}$, et al. Value of 3.0 T MR imaging in refractory partial epilepsy and negative 1.5 T MRI. Seizure 2010;19:475-8.

22. Phal PM, Usmanov A, Nesbit GM, Anderson JC, Spencer D, Wang P, et al. Qualitative comparison of 3-T and 1.5-T MRI in the evaluation of epilepsy. AJR Am J Roentgenol 2008;191:890-5.

23. Zijlmans M, de Kort GA, Witkamp TD, Huiskamp GM, Seppenwoolde $\mathrm{JH}$, van Huffelen AC, et al. $3 \mathrm{~T}$ versus $1.5 \mathrm{~T}$ phased-array MRI in the presurgical work-up of patients with partial epilepsy of uncertain focus. J Magn Reson Imaging 2009;30:256-62.

24. Knake S, Triantafyllou C, Wald LL, Wiggins G, Kirk GP, Larsson PG, et al. 3T phased array MRI improves the presurgical evaluation in focal epilepsies: a prospective study. Neurology 2005;65:1026-31.

25. De Ciantis A, Barkovich AJ, Cosottini M, Barba C, Montanaro D, Costagli $\mathrm{M}$, et al. Ultra-high-field MR imaging in polymicrogyria and epilepsy. AJNR Am J Neuroradiol 2015;36:309-16.

26. Oh BH, Moon HC, Baek HM, Lee YJ, Kim SW, Jeon YJ, et al. Comparison of $7 \mathrm{~T}$ and 3T MRI in patients with moyamoya disease. Magn Reson Imaging 2017;37:134-8.

27. Coras R, Milesi G, Zucca I, Mastropietro A, Scotti A, Figini M, et al. 7T MRI features in control human hippocampus and hippocampal sclerosis: an ex vivo study with histologic correlations. Epilepsia 2014;55:2003-16.

28. Lupo JM, Li Y, Hess CP, Nelson SJ. Advances in ultra-high field MRI for the clinical management of patients with brain tumors. Curr Opin Neurol 2011;24:605-15.

29. De Cocker LJ, Lindenholz A, Zwanenburg JJ, van der Kolk AG, Zwartbol M, Luijten PR, et al. Clinical vascular imaging in the brain at $7 \mathrm{~T}$. Neuroimage 2018;168:452-8.

30. Tallantyre EC, Dixon JE, Donaldson I, Owens T, Morgan PS, Morris PG, et al. Ultra-high-field imaging distinguishes MS lesions from asymptomatic white matter lesions. Neurology 2011;76:534-9.

31. De Ciantis A, Barba C, Tassi L, Cosottini M, Tosetti M, Costagli M, et al. 7T MRI in focal epilepsy with unrevealing conventional field strength imaging. Epilepsia 2016;57:445-54.

32. Veersema TJ, van Eijsden P, Gosselaar PH, Hendrikse J, Zwanenburg JJ, Spliet WG, et al. 7 tesla T2*-weighted MRI as a tool to improve detection of focal cortical dysplasia. Epileptic Disord 2016;18:315-23.

33. Balchandani P, Naidich TP. Ultra-high-field MR neuroimaging. AJNR Am J Neuroradiol 2015;36:1204-15.

34. Braakman HM, Vaessen MJ, Jansen JF, Debeij-van Hall MH, de Louw A, Hofman PA, et al. Pediatric frontal lobe epilepsy: white matter abnormalities and cognitive impairment. Acta Neurol Scand 2014;129:252-62.

35. Concha L, Beaulieu C, Collins DL, Gross DW. White-matter diffusion abnormalities in temporal-lobe epilepsy with and without mesial tem. poral sclerosis. J Neurol Neurosurg Psychiatry 2009;80:312-9.

36. Winston GP, Yogarajah M, Symms MR, McEvoy AW, Micallef C, Duncan JS. Diffusion tensor imaging tractography to visualize the relationship of the optic radiation to epileptogenic lesions prior to neurosurgery. Epilepsia 2011;52:1430-8.

37. Jeong JW, Asano E, Juhász C, Chugani HT. Quantification of primary motor pathways using diffusion MRI tractography and its application to predict postoperative motor deficits in children with focal epilepsy. Hum Brain Mapp 2014;35:3216-26.

38. Jeong JW, Asano E, Juhász C, Chugani HT. Localization of specific language pathways using diffusion-weighted imaging tractography for presurgical planning of children with intractable epilepsy. Epilepsia 2015; 56:49-57.

39. Hutchings F, Han CE, Keller SS, Weber B, Taylor PN, Kaiser M. Predicting surgery targets in temporal lobe epilepsy through structural connectome based simulations. PLoS Comput Biol 2015;11:e1004642.

40. Essayed WI, Zhang F, Unadkat P, Cosgrove GR, Golby AJ, O'Donnell LJ. White matter tractography for neurosurgical planning: a topographybased review of the current state of the art. Neuroimage Clin 2017;15: $659-72$.
41. Winston GP, Micallef C, Symms MR, Alexander DC, Duncan JS, Zhang $\mathrm{H}$. Advanced diffusion imaging sequences could aid assessing patients with focal cortical dysplasia and epilepsy. Epilepsy Res 2014;108:336-9.

42. Bruggemann JM, Wilke M, Som SS, Bye AM, Bleasel A, Lawson JA. Voxel-based morphometry in the detection of dysplasia and neoplasia in childhood epilepsy: combined grey/white matter analysis augments detection. Epilepsy Res 2007;77:93-101.

43. Colliot O, Bernasconi N, Khalili N, Antel SB, Naessens V, Bernasconi A. Individual voxel-based analysis of gray matter in focal cortical dysplasia. Neuroimage 2006;29:162-71.

44. Bonilha L, Montenegro MA, Rorden C, Castellano G, Guerreiro MM, Cendes F, et al. Voxel-based morphometry reveals excess gray matter concentration in patients with focal cortical dysplasia. Epilepsia 2006; 47:908-15.

45. Wagner J, Weber B, Urbach H, Elger CE, Huppertz HJ. Morphometric MRI analysis improves detection of focal cortical dysplasia type II. Brain 2011;134(Pt 10):2844-54.

46. Besson P, Bernasconi N, Colliot O, Evans A, Bernasconi A. Surface-based texture and morphological analysis detects subtle cortical dysplasia. Med Image Comput Comput Assist Interv 2008;11(Pt 1):645-52.

47. Briellmann RS, Syngeniotis A, Fleming S, Kalnins RM, Abbott DF, Jackson GD. Increased anterior temporal lobe T2 times in cases of hippocampal sclerosis: a multi-echo T2 relaxometry study at 3 T. AJNR Am J Neuroradiol 2004;25:389-94.

48. Winston GP, Vos SB, Burdett JL, Cardoso MJ, Ourselin S, Duncan JS. Automated T2 relaxometry of the hippocampus for temporal lobe epilepsy. Epilepsia 2017;58:1645-52.

49. Wang I, Alexopoulos A. MRI postprocessing in presurgical evaluation. Curr Opin Neurol 2016;29:168-74.

50. Hong SJ, Kim H, Schrader D, Bernasconi N, Bernhardt BC, Bernasconi A. Automated detection of cortical dysplasia type II in MRI-negative epilepsy. Neurology 2014;83:48-55.

51. Jin B, Krishnan B, Adler S, Wagstyl K, Hu W, Jones S, et al. Automated detection of focal cortical dysplasia type II with surface-based magnetic resonance imaging postprocessing and machine learning. Epilepsia 2018; 59:982-92.

52. Burneo JG, Poon R, Kellett S, Snead OC. The utility of positron emission tomography in epilepsy. Can J Neurol Sci 2015;42:360-71.

53. Vinton AB, Carne R, Hicks RJ, Desmond PM, Kilpatrick C, Kaye AH, et al. The extent of resection of FDG-PET hypometabolism relates to outcome of temporal lobectomy. Brain 2007;130(Pt2):548-60.

54. Knowlton RC, Elgavish RA, Bartolucci A, Ojha B, Limdi N, Blount J, et al. Functional imaging: II. Prediction of epilepsy surgery outcome. Ann Neurol 2008;64:35-41.

55. Tomás J, Pittau F, Hammers A, Bouvard S, Picard F, Vargas MI, et al. The predictive value of hypometabolism in focal epilepsy: a prospective study in surgical candidates. Eur J Nucl Med Mol Imaging 2019;46:1806-16.

56. Haller S, Zaharchuk G, Thomas DL, Lovblad KO, Barkhof F, Golay $\mathrm{X}$. Arterial spin labeling perfusion of the brain: emerging clinical applications. Radiology 2016;281:337-56.

57. Pendse N, Wissmeyer M, Altrichter S, Vargas M, Delavelle J, Viallon M, et al. Interictal arterial spin-labeling MRI perfusion in intractable epilepsy. J Neuroradiol 2010;37:60-3.

58. Blauwblomme T, Boddaert N, Chémaly N, Chiron C, Pages M, Varlet P, et al. Arterial Spin Labeling MRI: a step forward in non-invasive delineation of focal cortical dysplasia in children. Epilepsy Res 2014;108:1932-9.

59. Lee SM, Kwon S, Lee YJ. Diagnostic usefulness of arterial spin labeling in MR negative children with new onset seizures. Seizure 2019;65:151-8.

60. Sepeta LN, Berl MM, Wilke M, You X, Mehta M, Xu B, et al. Agedependent mesial temporal lobe lateralization in language fMRI. Epilep. sia 2016;57:122-30.

61. Collinge S, Prendergast G, Mayers ST, Marshall D, Siddell P, Neilly E, et al. Pre-surgical mapping of eloquent cortex for paediatric epilepsy surgery candidates: Evidence from a review of advanced functional neuroimaging. Seizure 2017:136-46.

62. Lee MH, Smyser CD, Shimony JS. Resting-state fMRI: a review of methods and clinical applications. AJNR Am J Neuroradiol 2013;34: 
1866-72.

63. Vadivelu S, Wolf VL, Bollo RJ, Wilfong A, Curry DJ. Resting-state functional MRI in pediatric epilepsy surgery. Pediatr Neurosurg 2013;49: 261-73.

64. Moeller F, Stephani U, Siniatchkin M. Simultaneous EEG and fMRI recordings (EEG-fMRI) in children with epilepsy. Epilepsia 2013;54:97182.

65. Nordli D, Xiao F, Zhou D. Real-time effects of centrotemporal spikes on cognition in rolandic epilepsy: An EEG-fMRI study. Neurology 2016; 87:552.

66. Negishi M, Martuzzi R, Novotny EJ, Spencer DD, Constable RT. Functional MRI connectivity as a predictor of the surgical outcome of epilepsy. Epilepsia 2011;52:1733-40.

67. Oishi M, Otsubo H, Kameyama S, Morota N, Masuda H, Kitayama M, et al. Epileptic spikes: magnetoencephalography versus simultaneous electrocorticography. Epilepsia 2002;43:1390-5.

68. Knowlton RC, Elgavish RA, Limdi N, Bartolucci A, Ojha B, Blount J, et al. Functional imaging: I. Relative predictive value of intracranial electroencephalography. Ann Neurol 2008;64:25-34.

69. Stefan H, Hummel C, Scheler G, Genow A, Druschky K, Tilz C, et al. Magnetic brain source imaging of focal epileptic activity: a synopsis of 455 cases. Brain 2003;126(Pt11):2396-405.

70. Aydin Ü, Vorwerk J, Dümpelmann M, Küpper P, Kugel H, Heers M, et al. Combined EEG/MEG can outperform single modality EEG or MEG source reconstruction in presurgical epilepsy diagnosis. PLoS One 2015;10:e0118753.

71. Bercovici E, Pang EW, Sharma R, Mohamed IS, Imai K, Fujimoto A, et al. Somatosensory-evoked fields on magnetoencephalography for epilepsy infants younger than 4 years with total intravenous anesthesia. Clin Neurophysiol 2008;119:1328-34. 